\title{
Research Paper \\ Direct and Indirect Measures of Attention Indicating a Bias Toward Cues in Methamphetamine Users
}

\author{
Seyed Davood Mirtorabi ${ }^{1}$ (D), Sharif Saleki² (D, Mohammad Sadegh Rahmanian ${ }^{3}$ (D), Hadi Hadizadeh ${ }^{4}$ (D), Reza Rostami ${ }^{5}$ (D), Ali Yoonessi ${ }^{*}$ (D) \\ 1. Department of Neurosciences and Addiction Studies, School of Advanced Technologies in Medicine, Tehran University of Medical Sciences, \\ Tehran, Iran \\ 2. Brain and Behavior Studies Laboratory, Tehran University of Medical Sciences, Iran. \\ 3. Legal Medicine Research Center, Legal Medicine Organization, Tehran, Iran. \\ 4. Department of Electrical Engineering, Faculty of Engineering, Quchan University of Technology, Quchan, Iran. \\ 5. Department of Psychology, Tehran University of Medical Sciences, Tehran, Iran
}

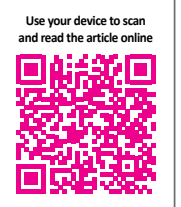

Citation Mirtorabi, S. D., Saleki, S., Rahmanian, M. S., Hadizadeh, H., Rostami, R., and Yoonessi, A. (2022). Direct and Indirect Measures of Attention Indicating a Bias Toward Cues in Methamphetamine Users. Basic and Clinical Neuroscience, 13(4), 455-464. http://dx.doi.org/10.32598/bcn.12.6.1589.1

ith http://dx.doi.org/10.32598/ben.12.6.1589.1

\section{(c) (1) (8)}

Article info:

Received: 10 Nov 2018

First Revision: 03 Jun 2019

Accepted: 06 Aug 2019

Available Online: $01 \mathrm{Jul} 2022$

Keywords:

Attentional bias,

Methamphetamine, Craving,

Substance addiction, Eye

movement, Measurements

\section{A B S T RA C T}

Introduction: This study aims to investigate the attentional bias toward drug-related stimuli along with subjective craving after encountering such stimuli in methamphetamine users. Studies of cue reactivity have confirmed a bias in attention and gaze toward drugrelated stimuli for most substances; however, methamphetamine drugs are less studied through a direct measure, such as eye tracking.

Methods: A total of 30 male subjects in the case group (methamphetamine users) and 36 subjects in the control group (no prior drug use) participated in this study. The participant's eye movement data were collected while they were viewing pairs of drug-related and nondrug images in a dot-probe paradigm. Craving was assessed via a self-report questionnaire on a scale of 0 to 10 before and after the psychophysical task.

Results: The analysis of eye-movement data showed a meaningful gaze bias toward cue images (drug-related) in the case group. Additionally, the gaze duration on cue images was significantly higher in the case group, in contrast to the control group. The same effect was observed in analyzing the dot-probe task; that is, the mean reaction time to a probe that replaced a cue image was significantly lower. The mean of the first-fixation measure in the control group was not significantly higher than chance; however, the percentage of the first-fixation on cue images in the drug users was meaningfully biased. Reported craving was significantly greater after performing the task compared to before.

Conclusion: Our results indicated an attentional bias toward drug-related cues in methamphetamine users as well as subjective craving after encountering such cues.

\section{* Corresponding Author:}

Ali Yoonessi, PhD.

Address: Department of Neurosciences and Addiction Studies, School of Advanced Technologies in Medicine, Tehran University of Medical Sciences, Tehran, Iran.

Tel: +98 (21) 8899118-23

E-mail: a_yoonessi@tums.ac.ir 


\section{Highlights}

- The gaze duration on cue images was significantly higher in methamphetamine users.

- The mean reaction time to a probe that replaced a cue image was significantly lower in methamphetamine users compared to the control group.

- The mean of the first-fixation measure in the case group was significantly better than chance.

- Craving was reported to be significantly greater after performing the task.

\section{Plain Language Summary}

Substance users tend to focus on the stimuli associated with substances. This is known as attention bias. Attention bias leads to increased craving. Attention bias for various substances has been previously reported; however, methamphetamine attention bias has not been evaluated so far. In this study, we measured the attention bias toward stimuli related to methamphetamine in methamphetamine users and control subjects with direct (eye tracking) and indirect (dot probe paradigm) methods. In addition, we measured the number of cravings in the case group. Our results confirmed the bias in attention toward methamphetamine-related stimuli in the case group compared to the control group.

\section{Introduction}

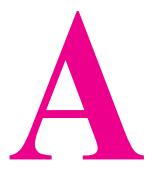

ddiction and regular drug use are often associated with responses to drug-related stimuli and cues (Carter \& Tiffany, 1999). For example, when alcoholics are presented with the sight or smell of an alcoholic beverage, or when smokers are asked to hold a lighted cigarette, they respond to the stimuli with increased physiological excitations and temptation which is referred to as craving.

Among addictive substances, amphetamine-type stimulants (ATS) considering that they can be manufactured almost anywhere are one of the most prevalent substances of abuse with 37 million users reported worldwide and reaching an all-time peak for a total seizures in recent years ("UNODC 2017," n.d.). About two-thirds of ATS users are in eastern and southeast Asia, and this substance has become their most frequent drug of abuse in some Asian countries.

Long-term use of methamphetamine can lead to $\operatorname{cog}$ nitive, intellectual, or affective disorders, which may persist long after discontinuation (Scott et al., 2007). Methamphetamine produces typical stimulatory effects, withdrawal symptoms (De La Garza, Shoptaw, \& Newton, 2008; McGregor et al., 2005; Newton et al., 2004), and craving (De La Garza et al., 2008) in many users. Methamphetamine users also elicit response inhibition deficiencies (Monterosso, Aron, Cordova, Xu, \& London, 2005). This suggests that impaired cognitive abili- ties may play a role in the use of methamphetamine drugs. Although the role of learning to respond to stimulants in methamphetamine users has not been clinically studied, pre-clinical studies have confirmed its effects (DerocheGamonet, Belin, \& Piazza, 2004; Everitt, Dickinson, \& Robbins, 2001; Vanderschuren \& Everitt, 2004).

\section{Craving}

Different definitions of craving have been given in the literature but it is usually referred to as the tendency to use an addictive substance. Other definitions, (BuydensBranchey, Branchey, Fergeson, Hudson, \& McKernin, 1997) considered deliberate substance use behavior as craving. Specifically, these researchers described craving for alcohol as "their chances of consuming alcohol if they are hospitalized and have unlimited financial resources." Marlatt (1985) suggested that the word "craving" tends to refer to the effects of the substance and that the term "urge" is considered a deliberate behavior of drug consumption. Other researchers have denoted "craving" for a wide range of phenomena, including the expectation of the positive effects of the substance and the desire and intention for using the substance (Tiffany \& Drobes, 1991). In some clinical trials, a craving for methamphetamine was associated with the usage dose (Hartz, Frederick-Osborne, \& Galloway, 2001).

Some researchers consider craving to have a relatively constant nature. Accordingly, people are asked to rate their cravings for a particular day or week (Anton, Moak, \& Latham, 1995). On the other hand, it is pos- 
sible to consider craving as a transient and recurrent phenomenon (often used in laboratory environments) (Shiffman, Paty, Gnys, Kassel, \& Hickcox, 1996). Momentary measurements confirm the view that craving even undergoes a fundamental change over a day, and expressing craving at different times has different meanings and predictive power (Shiffman et al., 1997).

Researchers also disagree on considering craving as a continuous structure that can be measured in a range of tendencies or limiting it to an experience that is characterized by intense tendency (West \& Schneiders, 1987). Some have suggested that craving may even exist in the absence of awareness (Berridge \& Robinson, 1995; Miller \& Gold, 1994). Accordingly, craving is a motivational structure that can be independent of conscious awareness. If an individual can experience craving without awareness (that is, an individual can differentiate between the craving process and the awareness of the craving), nonverbal measures of craving, such as searching and drug-using behaviors should be at least as notable as the measurements in self-report questionnaires of craving.

\section{Attention bias}

Cognitive biases of drug users brought about by drugrelated stimuli are an important aspect of drug cravings. Studying the eminent role of encountering such cues is essential for understanding the impact of these biases on drug seeking, craving, and relapse (Field \& Cox, 2008). Based on the proposed theories and models of addiction, it is plausible to assume that attentional bias and craving are two interrelated processes. Meanwhile, encountering drug-related cues can provoke at least two kinds of reactions in drug users: inclination toward attending to these cues, and craving (West, \& Jarvis, 2018). Recent models view attention and craving biases to be interconnected and mutually reinforced by each other with positive feedback cycles. Various indirect (addiction-Stroop task, dual task, flicker-induced change blindness, and visual probe task) and direct (such as eye-tracking and eventrelated potentials) methods have been used to measure attention bias in drug users (for a review see Field et al., 2009). In indirect methods, if the response time of a subject to the main task, while faced with a substance-related stimulus, is longer than when faced with a neutral stimulus, the results are interpreted with the assumption that a part of the individual's attention was involved with the substance-driven stimulus. Furthermore, in eye-tracking studies, attention can be measured when exposed to stimulus-dependent materials with greater precision and direct observation of behavior (i.e., eye movements are indicators of a shift in gaze). Stimulant drugs, such as methamphetamine and cocaine produce similar moodaltering and psychological symptoms (Mahoney et al., 2015) in users. Field et al. (2009) reviewed 5 studies in which attentional bias and craving of cocaine users were studied by direct and indirect measures. These researchers also found a weak correlation between subjective craving and attentional bias in 68 independent data sets, and even a weaker correlation was observed when they corrected for the publication bias.

This study aims to investigate the attentional bias toward drug-related stimuli and subjective craving after encountering such stimuli in methamphetamine users. We hypothesize that methamphetamine users elicit bias in attention and gaze toward drug-related images and their subjective experience of craving increases after encountering these cue images.

\section{Materials and Methods}

\section{Study participants}

A total of 40 methamphetamine users (all male) from a rehabilitation center participated in this study. Their last methamphetamine use was at least 8 and up to 29 days before the conduction of the study. The participants had not used any other drugs during the last 3 months. The control group consisted of 36 male subjects with no prior drug use.

All participants were evaluated to have normal vision. To detect any psychological disorders, a systematic psychological interview was performed by a psychiatrist according to the diagnosis criteria of DSM-5 (2013) on every subject. Furthermore, participants' urine samples were analyzed for every subject to determine their drug use. A total of 10 participants were removed from the study as 4 of them had psychological disorders (psychosis, depression, mood disorders, and so on) and 6 of them because of their positive urine analysis test. This process yielded a total number of 66 subjects (30 cases and 36 control) for this study. The study was registered in the Iranian Registry of Clinical Trials (Registration ID: IRCT201508038703N2) and all of the subjects signed an informed consent form indicating that they were aware of all conditions of this study.

\section{Study stimuli}

A total of 15 images related to methamphetamine were selected from images that were validated in previous studies (Ekhtiari, Alam-Mehrjerdi, Nouri, George, \& Mokri, 2010; 
Shahmohammadi et al., 2016). Each cue image was paired with a neutral image, and each pair was matched in semantic content and resolution as closely as possible. A total of 20 pairs of completely neutral images of natural scenes were randomly displayed for measuring any left-right bias. Each pair of images was displayed 4 times in such a way that the image associated with the amphetamines was placed twice on the right and twice on the left side of the screen. A total of 80 pairs of images ( 60 cue-neutral and 20 both neutral) were displayed to each subject for $1 \mathrm{~s}$. After displaying each image pair, a white spot (probe) randomly appeared on the right or left side of the monitor screen.

\section{Study procedure}

The task was presented in a low-light and low-noise room under no special environmental circumstances. The participants sat behind a desk at a distance of approximately $100 \mathrm{~cm}$ from a 17 -inch monitor while the eye tracker was set under it. After explaining the process of the experiment to the participants, they were presented with 10 pairs of images with the dot-probe task to ensure their understanding of the task (total run time of $5 \mathrm{~min}$ ). The images used in this step were not used in the main stage of the experiment and their data were not analyzed.

Subsequently, the eye tracker was calibrated for each participant. Eye movements were measured by the EyeTribe ET1000 eye-tracker with a $60 \mathrm{~Hz}$ sampling rate, which has been tested for spatial precision and accuracy in several studies (Dalmaijer, 2014; Ooms, Dupont, Lapon, \& Popelka, 2015). Before displaying each image, a fixation point $(+)$ was displayed at the center of the screen for 2000 milliseconds. The participants were asked to look at the fixation point and then the next image was presented.

After displaying each image pair, a probe randomly appeared on the right or left side of the monitor screen, and the participants specified the location of the probe by pressing one of the rights or left keys on the keyboard. Immediately after pressing the button, the probe disappeared, and the center probe was displayed again in the
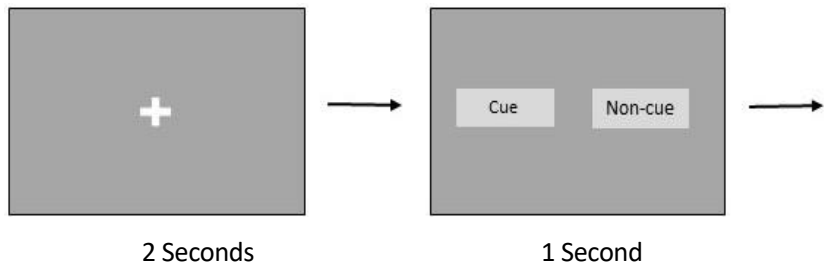

Figure 1. Order of displaying images to subjects middle of the screen, and then the next image was displayed for 1000 milliseconds (Figure 1).

In addition, the subjects in the drug user group were asked to declare their craving for methamphetamine via a self-reported craving questionnaire with a singleitem visual analog scale on a scale of 0 (not at all) to 10 (extremely craving). This was done once exactly before the start of the main task and once immediately after the completion of the task.

\section{Data acquisition}

The data was gathered using customized software developed for this study. The gaze direction was recorded (in degrees) every 17 milliseconds. If the eyes remained constant at a particular location for 100 milliseconds or more, that point was considered as a fixation and its duration was recorded. The eye movement data were analyzed in trials where cue and neutral images were displayed. The first fixation was found on cue or neutral images in these trails and the mean of the first fixation time was calculated separately for cue and non-cue images.

Furthermore, the percentage of the total fixation duration per image (dwell time) for each subject was calculated based on the total display time for each image. The difference between these percentages in neutral images and cues was considered as the attentional bias score for each individual.

In the data preparation phase for the dot-probe task, incorrect answers were excluded from further analysis. None of the participants had less than 50\% accuracy in the dot-probe task. The average response time was calculated individually for each subject, and the responses that had more absolute value than 3 Mean \pm SD from the mean, along with the response times below 200 milliseconds were discarded from each subject's data.

\section{Statistical analysis}

To determine the normality of each set of data, the Kolmogorov-Smirnov test, and the Shapiro Wilk test was used. The t-test was used to show the difference be-
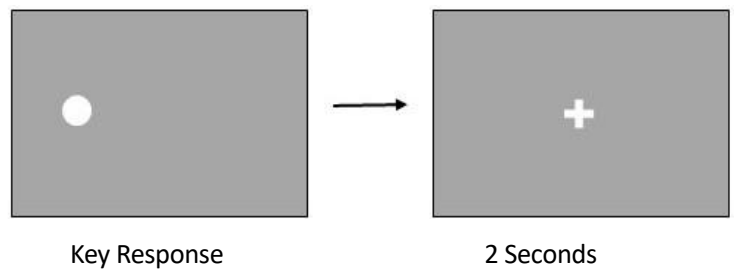

NEUR SCIENCE 
tween the eye movement data in each group and craving change in the methamphetamine group. For testing the difference in response time in the dot probe task, the Kruskal-Wallis test was used. Furthermore, to determine the difference between the groups, we used a 2-factor mixed analysis of variance (ANOVA). We used The Pearson correlation coefficient to illustrate the correlation between craving and attentional bias, age, and methamphetamine use variables.

\section{Results}

The case group included 30 men (Mean $\pm \mathrm{SD}$ age: $33.6 \pm 6.6$ years) who on average, 19.96 days had passed from their last methamphetamine use. The control group included 36 men who were matched for age with the case group (Mean \pm SD age: $33.7 \pm 6$ years). The participants' educational status is provided in Table 1.

Eye movement results

\section{First fixation duration}

to Perform a mixed design $2 \times 2$ ANOVA, the first fixation duration (Figure 2, left) for the type of image was used as the within-subject and group (case, control) as the between-subject variables. The results were significant for the group-image interaction $\left(\mathrm{F}_{1,64}=25.4\right.$, $\mathrm{P}<0.001$ ), and the analysis showed that drug-users (case group) had significantly longer fixation durations for cue images (Mean \pm SD: $192 \pm 37.4 \mathrm{~ms}$ ) than non-cue images (Mean \pm SD: $178 \pm 24.4 \mathrm{~ms})\left(\mathrm{t}_{29}=3.80, \mathrm{P}<0.001\right)$. However, in the control group, the mean duration of the first fixation on non-cue images (Mean \pm SD: $190 \pm 22.6 \mathrm{~ms}$ ) was significantly greater than cue images (Mean \pm SD: $183.5 \pm 29.7 \mathrm{~ms})\left(\mathrm{t}_{35}=3.11, \mathrm{P}=0.004\right)$.

\section{Dwell time}

Another ANOVA was performed for the mean dwell time percentage (Figure 2, right) with the same withinsubject (image-type) and between-subject (group) variables. Meaningful statistical interaction was observed for the image-type and group interaction $\left(\mathrm{F}_{1,64}=31.89\right.$, $\mathrm{P}<0.001)$. The mean dwell time percentage on cue and non-cue images for each participant was calculated concerning the total duration of stimulus presentation (1000 $\mathrm{ms}$ ). In the methamphetamine users, the eye gaze dwell time percentage on the cues (Mean \pm SD: $31 \pm 3.9$ ) was significantly longer than non-cues (Mean \pm SD: $26 \pm 3.3$ ) $\left(\mathrm{t}_{29}=6.15, \mathrm{P}<0.001\right)$. In the non-methamphetamine users, this percentage on cue images (Mean \pm SD: $31 \pm 4.5$ ) was significantly lesser than non-cue pictures (Mean $\pm \mathrm{SD}$ : $36 \pm 5.2)\left(\mathrm{t}_{35}=3.41, \mathrm{P}=0.002\right)$.

\section{Number of first fixations}

For each participant, the number of trials in which the first fixation was on the methamphetamine-related pictures was calculated as a proportion of the total number of trials in which the first fixation was on either the cue or the neutral image (Figure 3). In the case group, on average in $54.58 \% \pm 6.2 \%$ of the time first fixation occurred on cues, which was significantly greater than chance $\left(\mathrm{t}_{29}=3.987, \mathrm{P}<0.05,95 \%\right.$ CI $\left.[0.02,0.06]\right)$. In contrast, this proportion (Mean $\pm \mathrm{SD}: 50.6 \pm 0.055)$ did not entail a meaningful difference from chance in the control group.

Dot probe task results

We used the non-parametric Kruskal-Wallis test for cases that the probe appeared in place of the neutral image. To determine whether the average response time (Figure 4) of the drug-user group in the dot-probe task was different in cases where the probe appeared in the

Table 1. Educational status of the participants

\begin{tabular}{lccc}
\hline \multicolumn{2}{l}{ Variables } & \multicolumn{2}{c}{ No. (\%) } \\
\cline { 3 - 3 } Education & Under the diploma & Case & Control \\
\hline & Diploma & $6(20.0)$ & $6(16.7)$ \\
& Associate degree & $11(36.7)$ & $15(41.7)$ \\
& Bachelor & $6(20.0)$ & $6(16.7)$ \\
& Master's degree and higher & $5(16.7)$ & $3(8.3)$ \\
\hline
\end{tabular}


First Fixation

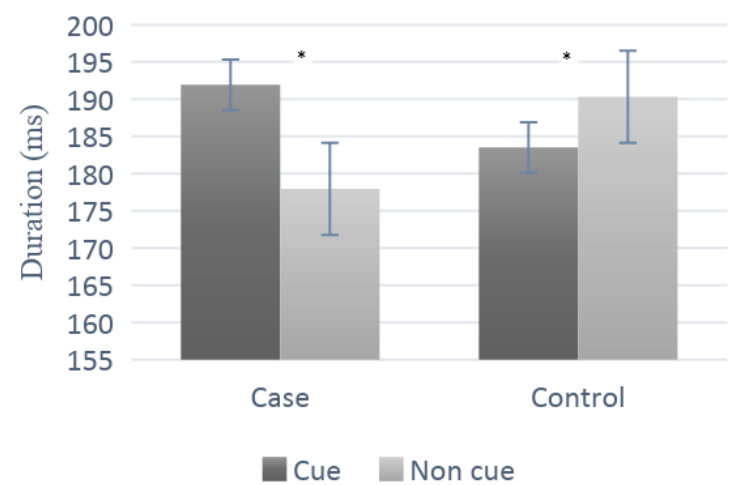

Dwell Time

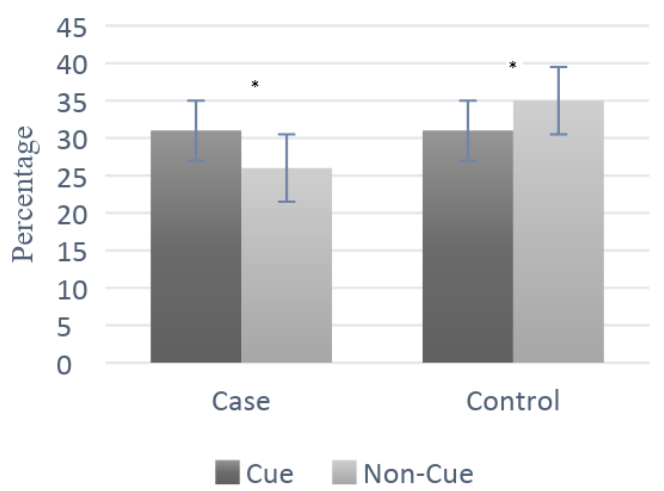

NEUR SCIENCE

Figure 2. Comparison between the duration of the first fixation and dwell time in the control group and the methamphetamine users groups

place of the cue image with the test (the Shapiro test showed the distribution of the sample is significantly not normal $[\mathrm{P}<0.001])$ which indicated a significant difference between the reaction time of the druguser group to these two probe placement conditions $(\mathrm{P}=0.033)$. The same analysis for the non-drug user group showed no statistical significance between the placement of the probe $(\mathrm{P}=0.376)$.

\section{Craving}

The reported craving after the task (Mean $\pm \mathrm{SD}$ : $4.23 \pm 2.029)$ was significantly greater than before the task (Mean \pm SD: $2.80 \pm 1.99)\left(\mathrm{t}_{29}=5.78, \mathrm{P}<0.001\right)$. The mean craving score had a negative correlation with the duration of the drug use $(\mathrm{r}=-0.3, \mathrm{P}=0.034)$, however, this correlation was small $\left(\mathrm{r}^{2}=0.10\right)$. The mean duration of the drug use of the case group was not significantly correlated with the reaction time variable $(\mathrm{P}=0.282)$. The analysis of the mean craving before the task and attentional bias (based on the percentage of mean gaze duration, first fixation, and reaction time) variables yielded no statistical significance.

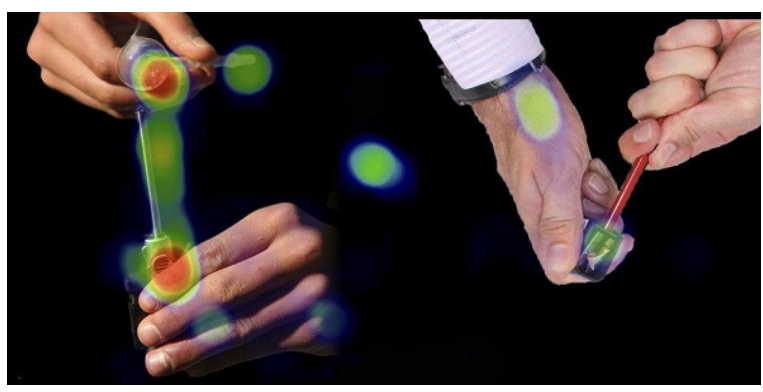

\section{Discussion}

Numerous studies previously indicated the attentional bias of cocaine, alcohol, opioids, and nicotine users; however, not many studies have focused on the effects of methamphetamine drugs on attentional bias and its relation to craving. In this study, we investigated the effects of methamphetamine abuse on attentional bias by employing direct and indirect methods for the measurement of such biases. The test results for the eye movement and the dotprobe paradigm showed consistent evidence of a bias toward cue images in methamphetamine users. The analysis of the eye tracking data demonstrated that drug users made more frequent first fixations on the cue images and looked at them for a longer period. In addition, the behavioral results showed that drug users had more vigilance toward cue images as they detected probes that replaced cue images faster. The dot-probe test shows a transient and limited aspect of attention, while the eye tracking data showed more dynamic and accurate visual search indicators. This shows that methamphetamine users have a significant bias toward drug-related images in various aspects of attention (LaBerge, Middleton, \& Wheatcroft, 1995), including primary shift and maintenance. These results are in line with a previous study that used the dual-task method to investigate

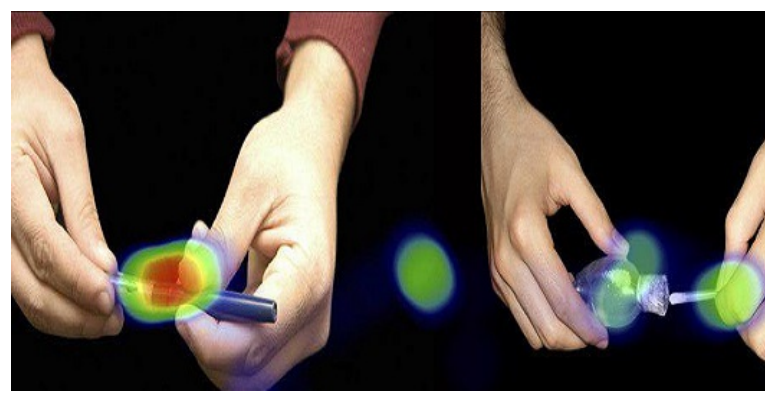

Figure 3. Sample heat maps generated from the eye tracking fixation data

NEUR OSCIENCE 


\section{Reaction Time in Dot Probe Task}

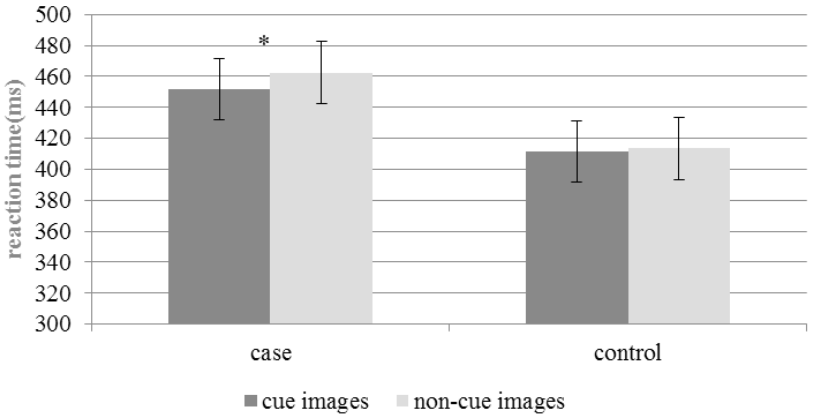

Figure 4. Comparison between average response times of the two groups

NEUR:SCIENCE

The probe appeared in place of the cue-image with the cases where the probe appeared in place of the non-cue image.

the attentional bias of methamphetamine users (Tolliver et al., 2012). In another study, Soleimannejad et al. (2015) examined morphine and methamphetamine users' responses toward emotional scenes during the early abstinence phase. They selected emotional and neutral stimuli that did not contain any cues to substances to avoid attentional bias because of craving and observed a similar pattern between methamphetamine users and the control group which indicated no bias toward pleasant or unpleasant images in methamphetamine users.

Although the rate of craving reported after the task was significantly higher than the reported craving before the task, there was no significant relationship between the degree of changes in craving with attentional bias in this study. Previous studies indicated conflicting results in this regard. Many studies have shown such a relationship in various drugs other than methamphetamine; however, in several studies, there is no significant relationship between craving and attentional bias (Ehrman et al., 2002; Field, 2006; Lubman, Peters, Mogg, Bradley, \& Deakin, 2000; Wertz \& Sayette, 2001). In some studies, where several methods for measuring attention bias have been employed, the craving was correlated with some of them (Mogg \& Bradley, 2002; Mogg, Bradley, Field, \& De Houwer, 2003).

The self-report measure was presented via a visual analog scale to measure the craving. Although the concept and the aim of this study were explained to the subjects with great care to reduce the defense mechanisms and the unrealistic expression of the craving - considering that the study was conducted in a rehabilitation center - the amount of craving reported might be biased. Theories of subjective craving and attentional bias predict a positive correlation between these two components; however, studies have found this correlation to be small (Field, Munafò, \& Franken, 2009). Perhaps one of the reasons for the lack of correlation between the rate of change of craving and the attentional bias in this study was the biased reported craving measure, considering the stigma of having a drug habit or relapsing after a period of clinical care. Further studies regarding the relationship between these two measures via more precise methods for the subjective craving will be helpful to shed light on this subject. A subjective measure of craving will in any way be accompanied by a bias; therefore, using objective methods can be more accurate.

The weak negative correlation between craving and the duration of drug use could be due to the aforementioned deficiencies in measuring craving, or they could indicate that methamphetamine craving is more aggressive in the earlier stages of addiction to this drug. More studies with more focus on the duration of methamphetamine use and its effects on the craving for this drug can determine which of these conclusions are true.

Some studies focus on the relationship between attention bias and relapse. The relationship between attention bias and change in addictive behavior seems to depend on the time interval between measuring attention bias and behavior change. The bias of attention may largely predict the likelihood of relapse shortly.

This study might imply diagnosis and determination of the rate of relapse or adherence to treatment. It is worth noting that there have been studies that have focused on changing the bias of attention in alcoholics and its impact on other drug-related indicators. This study can be a precursor to other studies on the manipulation of attention bias in methamphetamine users. 


\section{Ethical Considerations}

\section{Compliance with ethical guidelines}

Tehran University of Medical Sciences Ethics Committee approved the study and was registered in the Iranian Registry of Clinical Trials under the registration "ID IRCT201508038703N2." The protocol was approved by the Tehran University of Medical Sciences Ethics Committee. All subjects gave written informed consent according to the Declaration of Helsinki.

\section{Funding}

The paper was extracted from the $\mathrm{PhD}$. dissertation of the first author, Department of Neurosciences and Addiction Studies, School of Advanced Technologies in Medicine, Tehran University of Medical Sciences .

\section{Authors' contributions}

Conceptualization, Supervision: Ali Yoonessi, Reza Rostami, Hadi Hadizadeh; Methodology: Seyed Davood Mirtorabi, Sharif Saleki, Mohammad Sadegh Rahmanian; Investigation, Writing-review \& editing: All authors; Writing-original draft: Ali Yoonessi, Seyed Davood Mirtorabi, Sharif Saleki; Funding acquisition, Resources Ali Yoonessi; Seyed Davood Mirtorabi.

\section{Conflict of interest}

The authors declared no conflict of interest.

\section{References}

Anton, R. F., Moak, D. H., \& Latham, P. (1995). The Obsessive Compulsive Drinking Scale: A self-rated instrument for the quantification of thoughts about alcohol and drinking behavior. Alcoholism: Clinical and Experimental Research, 19(1), 92-99. [DOI:10.1111/j.1530-0277.1995.tb01475.x] [PMID]

Berridge, K. C., \& Robinson, T. E. (1995). The mind of an addicted brain: Neural sensitization of wanting versus liking. Current Directions in Psychological Science, 4(3), 71-75. [DOI:10.1111/1467-8721.ep10772316]

Buydens-Branchey, L., Branchey, M., Fergeson, P., Hudson J., \& McKernin, C. (1997). Hormonal, psychological, and alcohol craving changes after m-chlorophenylpiperazine administration in alcoholics. Alcoholism: Clinical and Experimental Research, 21(2), 220-227. [DOI:10.1111/j.1530-0277.1997. tb03753.x] [PMID]

Carter, B. L., \& Tiffany, S. T. (1999). Meta-analysis of cuereactivity in addiction research. Addiction, 94(3), 327-340. [DOI:10.1046/j.1360-0443.1999.9433273.x] [PMID]
Dalmaijer, E. (2014). Is the low-cost EyeTribe eye tracker any good for research? PeerJ PrePrints 2:e585v1. [DOI:10.7287/ peerj.preprints.585v1]

De La Garza, R., Shoptaw, S., Newton, T. F. (2008). Evaluation of the cardiovascular and subjective effects of rivastigmine in combination with methamphetamine in methamphetamine-dependent human volunteers. International Journal of Neuropsychopharmacology, 11(6), 729-741. [DOI:10.1017/ S1461145708008456] [PMID] [PMCID]

Deroche-Gamonet, V., Belin, D., \& Piazza, P. V. (2004). Evidence for addiction-like behavior in the rat. Science, 305(5686), 10141017. [DOI:10.1126/science.1099020] [PMID]

American Psychiatric Publishing (APA). (2013). Diagnostic and statistical manual of mental disorders: DSM-5. $5^{\text {th }}$ edition. Arlington, VA: American Psychiatric Publishing. [Link]

Ehrman, R. N., Robbins, S. J., Bromwell, M. A., Lankford, M. E., Monterosso, J. R., \& O'Brien, C. P. (2002). Comparing attentional bias to smoking cues in current smokers, former smokers, and non-smokers using a dot-probe task. Drug \& Alcohol Dependence, 67(2), 185-191. [DOI:10.1016/S03768716(02)00065-0]

Ekhtiari, H., Alam-Mehrjerdi, Z., Nouri, M., George, S., \& Mokri, A. (2010). Designing and evaluation of reliability and validity of visual cue-induced craving assessment task for methamphetamine smokers. Basic and Clinical Neuroscience, 1(4), 34-37. [Link]

Everitt, B. J., Dickinson, A., \& Robbins, T. W. (2001). The neuropsychological basis of addictive behaviour. Brain Research Reviews, 36(2-3), 129-138. [DOI:10.1016/S01650173(01)00088-1]

Field, M. (2006). Attentional biases in drug abuse and addiction: Cognitive mechanisms, causes, consequences and implications. In M. Munafò \& I. P. Albery (Eds.), Cognition and addiction (pp. 73-99). Oxford University Press. [Link]

Field, M., \& Cox, W. M. (2008). Attentional bias in addictive behaviors: A review of its development, causes, and consequences. Drug and Alcohol Dependence, 97(1-2), 1-20. [DOI:10.1016/j.drugalcdep.2008.03.030] [PMID]

Field, M., Munafò, M. R., \& Franken, I. H. (2009). A meta-analytic investigation of the relationship between attentional bias and subjective craving in substance abuse. Psychological Bulletin, 135(4), 589. [DOI:10.1037/a0015843] [PMID] [PMCID]

Hartz, D. T., Frederick-Osborne, S. L., \& Galloway, G. P. (2001). Craving predicts use during treatment for methamphetamine dependence: A prospective, repeated-measures, within-subject analysis. Drug \& Alcohol Dependence, 63(3), 269-276. [DOI:10.1016/S0376-8716(00)00217-9]

Laberge, S., Middleton, A. T., \& Wheatcroft, R. (1995). Characterization, nucleotide sequence, and conserved genomic locations of insertion sequence ISRm 5 in Rhizobium meliloti. Journal of bacteriology, 177(11), 3133-3142. [DOI:10.4159/harvard.9780674183940] [PMCID]

Lubman, D. I., Peters, L. A., Mogg, K., Bradley, B. P., \& Deakin, J. F. W. (2000). Attentional bias for drug cues in opiate dependence. Psychological Medicine, 30(1), 169-175. [DOI:10.1017/S0033291799001269] [PMID] 
Mahoney, J. J., 3rd, Thompson-Lake, D. G., Cooper, K., Verrico, C. D., Newton, T. F., \& De La Garza, R., 2nd (2015). A comparison of impulsivity, depressive symptoms, lifetime stress and sensation seeking in healthy controls versus participants with cocaine or methamphetamine use disorders. Journal of psychopharmacology (Oxford, England), 29(1), 50-56. [DOI:10.1177/0269881114560182] [PMID]

Marlatt, G.A. (1985) Cognitive factors in the relapse process In: Marlatt, G.A. and Gordon, J.R. Eds., Relapse Prevention, The Guilford Press, New York, 128-200. [Link]

McGregor, C., Srisurapanont, M., Jittiwutikarn, J., Laobhripatr, S., Wongtan, T., \& White, J. M. (2005). The nature, time course and severity of methamphetamine withdrawal. Addiction, 100(9), 1320-1329. [DOI:10.1111/j.13600443.2005.01160.x] [PMID]

Miller, N. S., \& Gold, M. S. (1994). Dissociation of "conscious desire" (craving) from and relapse in alcohol and cocaine dependence. Annals of Clinical Psychiatry, 6(2), 99-106. [DOI:10.3109/10401239409148988]

Mogg, K., \& Bradley, B. P. (2002). Selective processing of smoking-related cues in smokers: Manipulation of deprivation level and comparison of three measures of processing bias. Journal of Psychopharmacology, 16(4), 385-392. [DOI:10.1 177/026988110201600416] [PMID]

Mogg, K., Bradley, B. P., Field, M., \& De Houwer, J. (2003). Eye movements to smoking-related pictures in smokers: Relationship between attentional biases and implicit and explicit measures of stimulus valence. Addiction, 98(6), 825836. [DOI:10.1046/j.1360-0443.2003.00392.x] [PMID]

Monterosso, J. R., Aron, A. R., Cordova, X., Xu, J., \& London, E. D. (2005). Deficits in response inhibition associated with chronic methamphetamine abuse. Drug \& Alcohol Dependence, 79(2), 273-277. [DOI:10.1016/j.drugalcdep.2005.02.002] [PMID]

Newton, T. F., Kalechstein, A. D., Duran, S., Vansluis, N., \& Ling, W. (2004). Methamphetamine abstinence syndrome: preliminary findings. The American Journal on Addictions, 13(3), 248-255. [DOI:10.1080/10550490490459915] [PMID]

Ooms, K., Dupont, L., Lapon, L., \& Popelka, S. (2015). Accuracy and precision of fixation locations recorded with the lowcost Eye Tribe tracker in different experimental setups. Journal of Eye Movement Research, 8(1). [DOI:10.16910/jemr.8.1.5]

Scott, J. C., Woods, S. P., Matt, G. E., Meyer, R. A., Heaton, R. K., Atkinson, J. H., \& Grant, I. (2007). Neurocognitive effects of methamphetamine: A critical review and meta-analysis. Neuropsychology Review, 17(3), 275-297. [DOI:10.1007/ s11065-007-9031-0] [PMID]

Shahmohammadi, F., Golesorkhi, M., Riahi Kashani, M. M., Sangi, M., Yoonessi, A., \& Yoonessi, A. (2016). Neural correlates of craving in methamphetamine abuse. Basic and Clinical Neuroscience, 7(3), 221-230. [DOI:10.15412/J. BCN.03070307] [PMID] [PMCID]

Shiffman, S., Engberg, J. B., Paty, J. A., Perz, W. G., Gnys, M., Kassel, J. D., et al. (1997). A day at a time: Predicting smoking lapse from daily urge. Journal of Abnormal Psychology, 106(1), 104. [DOI:10.1037/0021-843X.106.1.104] [PMID]

Shiffman, S., Paty, J. A., Gnys, M., Kassel, J. A., \& Hickcox, M. (1996). First lapses to smoking: within-subjects analysis of real-time reports. Journal of Consulting and Clinical Psychology, 64(2), 366. [DOI:10.1037/0022-006X.64.2.366] [PMID]
Shahmohammadi, F., Golesorkhi, M., Riahi Kashani, M. M. Sangi, M., Yoonessi, A., \& Yoonessi, A. (2016). Neural correlates of craving in methamphetamine abuse. Basic and Clinical Neuroscience, 7(3), 221-230. [DOI:10.15412/J. BCN.03070307] [PMID] [PMCID]

Soleimannejad, M., Tehrani-Doost, M., Khorrami, A., Joghataei, M. T., \& Pishyareh, E. (2015). Evaluation of attention bias in morphine and methamphetamine abusers towards emotional scenes during early abstinence: An eye-tracking study. Basic and clinical neuroscience, 6(4), 223-230.

Tiffany, S. T., \& Drobes, D. J. (1991). The development and initial validation of a questionnaire on smoking urges. $A d-$ diction, 86(11), 1467-1476. [DOI:10.1111/j.1360-0443.1991. tb01732.x] [PMID]

Tolliver, B. K., Price, K. L., Baker, N. L., LaRowe, S. D., Simpson, A. N., McRae-Clark, A. L., et al. (2012). Impaired cognitive performance in subjects with methamphetamine dependence during exposure to neutral versus methamphetamine-related cues. The American Journal of Drug and Alcohol Abuse, 38(3), 251-259. [DOI:10.3109/00952990.2011.6 44000] [PMID]

UNODC. World Drug Report. (2017). Pre-briefing to the Member States. Vienna, 16 June 2017. Retrieved from: [Link]

Vanderschuren, L. J., \& Everitt, B. J. (2004). Drug seeking becomes compulsive after prolonged cocaine self-administration. Science, 305(5686), 1017-1019. [DOI:10.1126/science.1098975] [PMID]

Wertz, J. M., \& Sayette, M. A. (2001). Effects of smoking opportunity on attentional bias in smokers. Psychology of Addictive Behaviors, 15(3), 268. [DOI:10.1037/0893-164X.15.3.268] [PMID] [PMCID]

West, R., \& Jarvis, M. J. (2018). Is 'hardcore smoker' a useful term in tobacco control? Addiction (Abingdon, England), 113(1), 3-4. [DOI:10.1046/j.1360-0443.2001.96131.x] [PMID]

West, R., \& Schneider, N. (1987). Craving for cigarettes. British Journal of Addiction, 82(4), 407-415. [DOI:10.1111/j.1360-0443.1987.tb01496.x] [PMID] 
This Page Intentionally Left Blank 